\title{
MicroRNA-145 regulates the differentiation of human adipose-derived stem cells to smooth muscle cells via targeting Krüppel-like factor 4
}

\author{
KAISAIER AJI $^{1 *}$, YUN ZHANG $^{2 *}$, ABUDUSAIMI AIMAITI $^{3}$, YUJIE WANG ${ }^{1}$, \\ MULATI REXIATI $^{1}$, BAIHETIYA AZHATI ${ }^{1}$, HAMULATI TUSONG ${ }^{1}$, LEI CUI ${ }^{1,4}$ and CHEN WANG ${ }^{5}$
}

\author{
${ }^{1}$ Department of Urology, The First Affiliated Hospital of Xinjiang Medical University, Urumqi, Xinjiang 830054; \\ ${ }^{2}$ Department of Orthopedics, Tongji University School of Medicine, Shanghai 200092; ${ }^{3}$ Department of Joint Surgery, \\ The First Affiliated Hospital of Xinjiang Medical University, Urumqi, Xinjiang 830054; ${ }^{4}$ Medical Technology \\ and Engineering Institute, Henan Science and Technology University, Luoyang, Henan 471023; \\ ${ }^{5}$ Department of Plastic and Reconstructive Surgery, Shanghai 9th People's Hospital, Shanghai \\ Jiao Tong University School of Medicine, Shanghai 200011, P.R. China
}

Received November 18, 2015; Accepted November 4, 2016

DOI: $10.3892 / \mathrm{mmr} .2017 .6478$

\begin{abstract}
Understanding the molecular mechanisms underlying human adipose-derived stem cell (hASC) differentiation to smooth muscle may contribute to the development of effective therapies for relevant muscle defects, such as bladder wall and urethral defects. A previous study described the differentiation of hASCs to smooth muscle cells (SMCs) by transforming growth factor- $\beta 1$ (TGF- $\beta 1$ ) and bone morphogenetic protein- 4 (BMP4) treatment. The present study investigated whether microRNA-145 (miR-145) may be involved in the process of hASC differentiation. The expression of miR-145 was significantly increased during differentiation of ASCs to SMCs. SMC-specific genes and proteins, including a-smooth muscle actin ( $\alpha$-SMA), smooth muscle protein-22 $\alpha($ SM22 $\alpha)$, calponin and myosin heavy chain (SM-MHC) were upregulated by transfection of a miR-145 mimic. By contrast, these factors were downregulated following introduction of antisense oligonucleotides. In addition, Krüppel-like factor 4 (KLF4) levels, which decreased during the differentiation of hASCs,
\end{abstract}

Correspondence to: Dr Lei Cui, Department of Urology, The First Affiliated Hospital of Xinjiang Medical University, 317 South Liyushan Road, Urumqi, Xinjiang 830054, P.R. China E-mail: cuileijy@163.com

Dr Chen Wang, Department of Plastic and Reconstructive Surgery, Shanghai 9th People's Hospital, Shanghai Jiao Tong University School of Medicine, 227 South Chongqing Road, Shanghai 200011, P.R. China

E-mail: wangchen2369@163.com

${ }^{*}$ Contributed equally

Key words: adipose-derived stem cell, microRNA-145, smooth muscle differentiation, Krüppel-like factor 4 were downregulated when the cells were transfected miR-145 mimics. Futhermore, inhibition of KLF4 by treatment with short-interfering-RNA against KLF4, resulted in increased expression of SMC-specific genes and proteins. In conclusion, the results of the present study demonstrated that by regulating KLF4, miR-145 may be involved in regulating smooth muscle differentiation of ASCs induced by TGF- $\beta 1$ and BMP4.

\section{Introduction}

Smooth muscle is a major component of human tissues and is essential for the normal function of many organs, including the intestines, urinary tract and vascular system (1). Smooth muscle tissue defects or damage caused by congenital or acquired abnormalities may result in severe dysfunctions. Tissue engineering and regenerative medicine may be used to repair these defects using seed cells and biomaterials, including smooth muscle cells (SMCs) (2), bone marrow stem cells (3) and adipose-derived stem cells (ASCs) (4). However, mature differentiated SMCs demonstrate a limited ability to proliferate, and usually lose their contractile phenotype and convert to a synthetic phenotype during in vitro expansion (5). Therefore, further investigation into alternative cell sources for blood vessel engineering is required, as a large number of functional cell types are usually involved in this process (6).

Adipose tissue consists of an abundance of mesenchymal stem cells (MSCs, known as ASCs), with faster growth and higher proliferative capacities when compared with MSCs from other sources (7). In addition, the multipotency of ASCs is independent of the age of the donor $(8,9)$. ASCs demonstrate the potential to differentiate into osteocytes (10), neural cells (11) and muscular cells (12).

ASCs express smooth muscle-specific contractile proteins when stimulated by transforming growth factor- $\beta 1$ (TGF- $\beta 1$ ) and bone morphogenetic protein-4 (BMP4), and the differentiated cells exhibit levels of contractility similar to that of SMCs (13). TGF- $\beta 1$ and BMP4 are potent inducers of genes 
involved in contractility (14). Transcription of contractile genes is positively regulated by a regulatory DNA element known as the CArG box (15). The CArG box is activated by binding of the serum response factor (SRF) together with its coactivators myocardin (MYOCD) and myocardin-related transcription factors (MRTFs) (16-18). Krüppel-like factor 4 (KLF4) inhibits activation of the CArG box (19). These observations suggest that modulation of KLF4 may be a prerequisite for the induction of contractile genes by TGF-b1 and BMP4 (20).

MicroRNAs (miRNAs) are a group of post-transcriptional regulators that serve a major role in a number of diverse functions, including cell proliferation, apoptosis and organogenesis (21). In addition, miRNAs are regulators of the differentiation, self-renewal and division of stem cells $(22,23)$. Previous studies have identified an important role of miR-145 in mechanisms of smooth muscle differentiation and function, including the direct and indirect effects of miRNA-145 on MYOCD expression $(24,25)$, angiotensin signaling (26) and actin polymerization (27). Cheng et al (25) demonstrated that overexpression of miR-145 increased the expression of vascular smooth muscle cell (VSMC) differentiation marker genes, including smooth muscle $\alpha$-actin, calponin and smooth muscle-myosin heavy chain (SM-MHC). The levels of these marker genes were decreased in cultured VSMCs following treatment with a miR-145 inhibitor. In addition to regulating VSMC differentiation markers, miR-145 alone was able to maintain the differentiated spindle-like shape and inhibit VSMC proliferation.

The present study investigated human ASCs treated with TGF- $\beta 1$ and BMP4 following transfection with miR-145 mimics or antisense oligonucleotides. Expression alterations in smooth muscle contractile proteins and their associated genes were examined to investigate the effects of miR-145. Short-interfering RNA (si-RNA) targeting KLF4 was used to mimic the differentiation of ASCs to SMCs by TGF- $\beta 1$ and BMP4. This aimed to further clarify the role of miR-145 in the differentiation process.

\section{Material and methods}

Isolation and culture of human ASCs (hASCs). Fresh human lipoaspirate fractions were obtained from 12 donors who had received abdominal liposuction (4 male, 8 female; age, 25-52 years; weight, $82-97 \mathrm{~kg}$ ). The donors were admitted by the Research Ethical Committee of The First Affiliated Hospital of Xinjiang Medical University (Urumqi, China) and were admitted to the same hospital in 2013. All donors had provided written informed consent for the use of their samples in the present study. The study was approved by the Research Ethical Committee of The First Affiliated Hospital of Xinjiang Medical University (Urumqi, China). Fresh lipoaspirate fractions were washed with phosphate-buffered saline (PBS) and treated with $0.075 \%$ type I collagenase (Sigma-Aldrich; Merck Millipore, Darmstadt, Germany) under shaking (120 rpm) at $37^{\circ} \mathrm{C}$ for $60 \mathrm{~min}$. The enzyme activity was neutralized with low-glucose Dulbecco's Modified Eagle's Medium (LG-DMEM; Gibco; Thermo Fisher Scientific, Inc., Waltham, MA, USA) containing 10\% fetal bovine serum (FBS; Hylone; GE Healthcare Life Sciences, Logan, UT, USA). The digested lipoaspirate samples were then centrifuged at 1,200 $\mathrm{xg}$ for
$10 \mathrm{~min}$ at $20^{\circ} \mathrm{C}$ to obtain the high density stromal vesicular fraction, which was filtered through a $50 \mu \mathrm{m}$ nylon mesh to remove undigested tissue, and subsequently centrifuged at $1,000 \mathrm{xg}$ for $10 \mathrm{~min}$ at $20^{\circ} \mathrm{C}$. The supernatant was discarded and the pellet was resuspended in LG-DMEM supplemented with $10 \%$ FBS, $100 \mathrm{U} / \mathrm{ml}$ penicillin (Sigma-Aldrich; Merck Millipore) and $100 \mathrm{mg} / \mathrm{ml}$ penicillin/streptomycin (Sigma-Aldrich; Merck Millipore). The cells were seeded in 100-mm culture dishes at a density of $4 \times 10^{4}$ cells $/ \mathrm{cm}^{2}$ and the medium was refreshed twice each week. When cells reached $70-80 \%$ confluence, they were passaged and cells from passage 3 to 5 were used for the purposes of this study. The ability of hASCs to differentiate into osteogenic, adipogenic and chondrogenic lineages was examined as previously reported (8).

Induction of SMC differentiation. The hASCs were induced to differentiate into SMCs using TGF- $\beta 1$ and BMP4 (R\&D Systems, Inc., Minneapolis, MN, USA) following serum starvation. The differentiation medium consisted of $5 \mathrm{ng} / \mathrm{ml}$ TGF- $\beta 1$, $2.5 \mathrm{ng} / \mathrm{ml} \mathrm{BMP} 4$ and $1 \% \mathrm{FBS}$. The media was refreshed every 2 days. Cell characterization and functional evaluation was performed following 7 days of culture.

miRNA mimic and antisense oligonucleotide transfection. The miRNA mimics, antisense oligonucleotides and negative control were synthesized by Guangzhou RiboBio Co., Ltd. (Guangzhou, China). The miR-145 and anti-miR-145 oligonucleotides were designed according to the miRBase sequence database (mirbase.org) (28). The sequence of the miR-145 mimic was 5'-GUCCAGUUUUCCCAGGAAUCCCU-3'; and the anti-miR-145 sequence was 5'-AGGGAUUCCUGGGAA AACUGGAC-3'. A random sequence was used as the negative control: 5'-CGGCGGTTGAGATGAAGCACTG-3'.

The ASCs were seeded onto a 24-well culture plate at a density of $1 \times 10^{5}$ cells $/ \mathrm{cm}^{2}$ at $24 \mathrm{~h}$ prior to transfection. Cells were transfected when cells reached $80 \%$ confluency. The miR-145 mimics, miR-145 inhibitor and negative controls were diluted using $30 \mu \mathrm{l} 1 \mathrm{X}$ riboFECT ${ }^{\mathrm{Tu}} \mathrm{CP}$ Buffer (Guangzhou RiboBio Co., Ltd.) and incubated at room temperature for 5 min. A total of $3 \mu \mathrm{l}$ riboFECT ${ }^{\mathrm{Tt}} \mathrm{CP}$ Reagent (Guangzhou RiboBio Co., Ltd.) was added and incubated at room temperature for $15 \mathrm{~min}$. riboFECT ${ }^{\text {Ti }} \mathrm{CP}$ mixture (Guangzhou RiboBio Co., Ltd.) was added to $465.75 \mu \mathrm{l}$ serum-free, cell culture medium without penicillin/streptomycin. All cells were cultured for $6 \mathrm{~h}$ with above conditions. Culture medium was then changed to growth medium (DMEM with 10\% FBS without penicillin/streptomycin). Cells were harvested $48 \mathrm{~h}$ following transfection and were then analyzed.

Reverse transcription-quantitative polymerase chain reaction $(R T-q P C R)$. For RT-qPCR analysis, RNA was extracted using TRIzol Reagent (Invitrogen; Thermo Fisher Scientific, Inc.) according to the manufacturer's instructions, and cDNA was synthesized using PrimeScript ${ }^{\mathrm{TM}}$ RT Master Mix (Takara Bio, Inc., Otsu, Japan). A total of $1 \mu$ l total RNA was reverse transcribed into cDNA. The 5X PrimerScript master mix (Takara Bio, Inc.) was used to reverse transcribe RNA. The reactions were performed and monitored in a Biometra T3 thermocycler (Biometra GmbH, Göttingen, Germany). qPCR was performed using a 7500 Fast Real Time PCR system (Applied 
Biosystems; Thermo Fisher Scientific, Inc.) with thermocycling parameters consisting of $94^{\circ} \mathrm{C}$ for $3 \mathrm{~min}$, followed by 40 cycles of $94^{\circ} \mathrm{C}$ for $15 \mathrm{sec}$ and $60^{\circ} \mathrm{C}$ for $40 \mathrm{sec}$, according to the manufacturer's protocol. SYBR Premix Ex Taq (Takara Bio, Inc.) was used in each reaction. The PCR primers were as follows: a-smooth muscle actin ( $\alpha$-SMA), forward, 5'-GGT GATGGTGGGAATGGG-3' and reverse, 5'-GCAGGGTGG GATGCTCTT-3'; smooth muscle protein-22 $\alpha$ (SM22 $\alpha$ ), forward, 5'-AACAGCCTGTACCCTGATGG-3' and reverse, 5'-CGGTAGTGCCCATCATTCTT-3'; Calponin, forward, 5'-ATGTCCTCTGCTCACTTCA-3' and reverse, 5'-TTTCCG CTCCTGCTTCTCT-3'; SM-MHC, forward, 5'-TGCTTT CGCTCGTCTTCC-3' and reverse, 5'-CGGCAACTCGTG TCCAAC-3'; KLF4, forward, 5'-CCCAATTACCCATCCTTC CT-3' and reverse, 5'-CGTCCCAGTCACAGTGGTAA-3'; miR-145, forward, 5'-CGGCGGTGTCCAGTTTTCCCA GGA-3' and reverse, 5'-GTCGTATCCAGTGCAGGGTCC GAGGTATTCGCACTGGATACGACAGGGAT-3'. $\beta$-actin forward, 5'-ATCATGTTTGAGACCTTCAA-3' and reverse, 5'-CATCTCTTGCTCGAAGTCCA-3'; U6 forward, 5'-CTC GCTTCGGCAGCACA-3' and reverse, 5'-AACGCTTCACGA ATTTGCGT-3'. All experiments were performed in triplicate. The relative expression of target mRNA was normalized to the expression of $\beta$-actin. The expression level of miR-145 was normalized to U6 and the fold-change was calculated using the $2^{-\Delta \Delta \mathrm{Cq}}$ method of relative quantification (29).

Western blotting. Cells were harvested and lysed in radioimmunoprecipitation buffer with added protease and phosphatase inhibitors (Roche Diagnostics, Indianapolis, IN, USA). The protein concentration was determined using the bicinchoninic acid method (Bio-Rad Laboratories, Inc. Hercules, CA, USA). Total protein ( 20-50 $\mu \mathrm{g})$ was separated using a $10 \%$ SDS-PAGE gel, before it was transferred to polyvinylidene difluoride membranes. Membranes were blocked in Tris-buffered saline containing $0.05 \%$ Tween-20 (Sigma-Aldrich; Merck Millipore) and 5\% skim milk, and then incubated with the following primary antibodies from Abcam (Cambridge, UK): Rabbit polyclonal to SMA (cat. no, ab5694; dilution, 1:1,000), rabbit polyclonal anti-SM22 $\alpha$ (cat. no, ab14106; dilution, 1:1,000), rabbit monoclonal anti-calponin (cat. no, ab46794; dilution, 1:20,000) rabbit polyclonal to SM-MHC (cat. no, ab53219; dilution, 1:1,000), rabbit monoclonal anti-KLF4 (cat. no, ab151733; dilution, 1:1,000) and GAPDH (cat. no, ab181603; dilution, 1:2,000). Membranes were then washed with Tris- $\mathrm{HCl}$ with Tween-20 (TBST; Sigma Aldrich; Merck-Millipore), incubated with the secondary antibody (horseradish peroxidase-conjugated goat anti-rabbit antibody, dilution, 1:2,000; AP307P; EMD Millipore, Billerica, MA, USA) at room temperature for $2 \mathrm{~h}$ and detected using enhanced chemiluminescence (Bio-Rad Laboratories, Inc.). GAPDH was used as an internal loading control.

RNA interference. As a negative control, a non-targeting scrambled siRNA (Shanghai Usen Biotechnology, Shanghai, China) was used. The sequence of the KLF4 siRNA was 5'-GGACGGCUGUGGAUGGAAATT-3' (Shanghai Usen Biotechnology). The hASCs were seeded into 24-well plates at density of $1 \times 10^{5}$ cells $/ \mathrm{cm}^{2}$ and transfected with $100 \mathrm{pmol} /$ well
siRNA using Lipofectamine 2000 (Invitrogen; Thermo Fisher Scientific, Inc.), according to the manufacturer's instructions. The medium was refreshed following $6 \mathrm{~h}$ transfection, and the cells were harvested $48 \mathrm{~h}$ following transfection for total RNA and protein isolation.

Immunofluorescence staining. Immunofluorescence was performed on methanol-fixed cells (density, $1 \times 10^{5} \mathrm{cells} / \mathrm{cm}^{2}$; 4\% methanol) using the following primary antibodies purchased from Abcam: Rabbit polyclonal anti- $\alpha$-SMA (dilution, 1:100; cat. no. ab5694), rabbit polyclonal anti-SM22 $\alpha$ (dilution, 1:250; cat. no. ab14106), rabbit monoclonal anti-calponin (dilution, 1:150; cat. no. ab46794) and rabbit polyclonal anti-SM-MHC (dilution, 1:50; cat. no. ab53219). Following incubation with primary antibodies for $60 \mathrm{~min}$ at room temperature, the cells were washed with PBS three times. The Alexa Fluor 594-conjugated donkey anti-rabbit secondary antibody (dilution, 1:5,000; cat. no. R37119; Thermo Fisher Scientific, Inc.) was used to detect the localization of anti- $\alpha$-SMA, anti-SM22 $\alpha$, anti-calponin and anti-SM-MHC antibodies. Cell nuclei were stained with DAPI. The images were visualized using a confocal laser scanning platform microscope (TCS SP8; Leica Microsystems GmbH, Wetzlar, Germany).

Luciferase reporter assay. The wild-type 3'-untranslated (UTR) regions of KLF-4 (nt=549-1988) and corresponding mutations were amplified using PCR, followed by cloning into a pLVX report vector (Thermo Fisher Scientific, Inc.). 293T cells (Shanghai Institutes for Biological Sciences, Chinese Academy of Sciences, Shanghai, China) were seeded onto 24-well plates at a density of $1 \times 10^{5} / \mathrm{cm}^{2}$. When the cell confluency reached $70-80 \%$, the reporter gene plasmid and miR-145 were co-transfected into 293T cells by using Lipofectamine 2000 (Thermo Fisher Scientific, Inc.). Following $30 \mathrm{~h}$ after transfection, cells were collected and luciferase activity was detected. The experiment was repeated in triplicate.

Statistical analysis. Data are expressed as mean \pm standard deviation from at least three independent experiments. Statistical analysis was performed using Student's t-test using SPSS software (version, 17.0; SPSS Inc., Chicago, IL, USA). $\mathrm{P}<0.05$ was considered to indicate a statistically significant difference.

\section{Results}

hASCs differentiate into SMCs when treated with TGF- $\beta 1$ and $B M P 4$. In preliminary experiments, hASCs were successfully induced into SMCs. The hASCs exhibited a fine, elongated, fibroblast-like morphology when subcultured in normal medium. Cells at passages 3-5 stimulated by TGF- $\beta 1$ and BMP4 for 7 days demonstrated a spindle-like morphology and proliferated at fluctuating rates (data not shown). To confirm whether hASCs differentiated into SMCs when treated with TGF- $\beta 1$ and BMP4, mRNA levels of smooth muscle-specific contractile proteins $\alpha$-SMA, SM22 $\alpha$, calponin and SM-MHC were detected by RT-qPCR analysis. The mRNA levels of these markers were significantly increased 7 days following differentiation ( $\alpha$-SMA, $\mathrm{P}=0.032$; SM22 $\alpha, \mathrm{P}=0.041$; calponin, $\mathrm{P}=0.018$; SM-MHC, $\mathrm{P}=0.027$ ) (Fig. 1A and $\mathrm{B}$ ). To investigate 
A

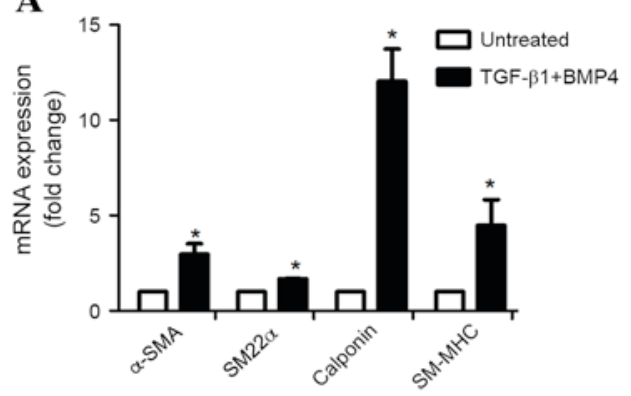

C

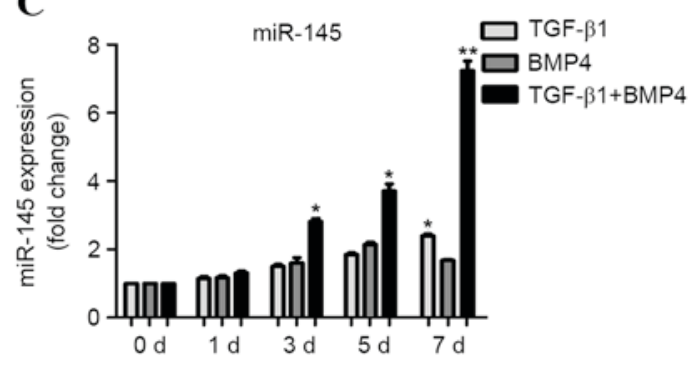

B

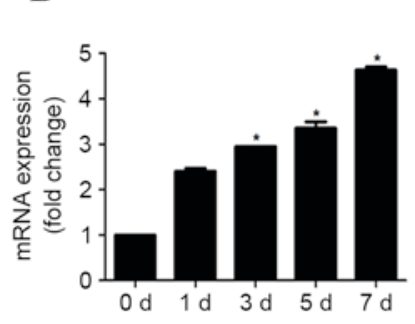

$\operatorname{SM} 22 \alpha$

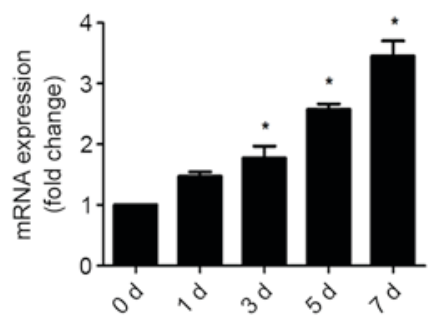

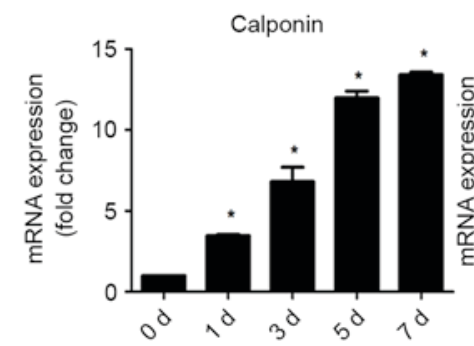

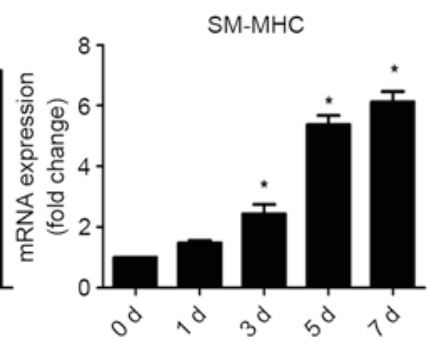

Figure 1. Smooth muscle differentiation of hASCs by TGF- $\beta 1$ and BMP4. The mRNA expression levels of smooth muscle-specific markers and miR-145 were measured by reverse transcription-quantitative polymerase chain reaction. (A) The mRNA levels of smooth muscle-specific markers significantly increased following 7 days of differentiation. (B) The expression of the smooth muscle-specific genes at different time points following treatment with TGF- $\beta 1$ and BMP4. (C) miR-145 levels increased gradually during the differentiation of hASCs into smooth muscle cells. Data are presented as the mean \pm standard deviation $(\mathrm{n}=3)$. ${ }^{*} \mathrm{P}<0.05$ and ${ }^{* *} \mathrm{P}<0.01$ vs. day 0 . hASCs, human adipose-derived stem cells; TGF- $\beta 1$, transforming growth factor $\beta 1$; BMP4, bone morphogenetic protein 4; miR-145, microRNA-145; $\alpha$-SMA, $\alpha$-smooth muscle actin; SM22 $\alpha$, smooth muscle protein-22 $\alpha$; SM-MHC, smooth muscle-myosin heavy chain.

the role of miR-145 in SMC differentiation, miR-145 expression levels were detected by RT-qPCR on day $0,1,3$, 5 and 7 following differentiation. The expression of miR-145 significantly increased at day 3, 5 and 7 following induction of differentiation with TGF- $\beta 1$ and BMP4 (3d, P=0.019; $5 \mathrm{~d}$, $\mathrm{P}=0.011 ; 7$ d, $\mathrm{P}=0.002$; Fig. 1C).

Overexpression of miR-145 levels in hASCs undergoing smooth muscle cell differentiation. For functional evaluation of miR-145, hASCs were transfected with miR-145 mimics, their counterpart inhibitor (miR-control), anti-miR-145 or a negative control (anti-NC). Quantification of miR-145 expression levels by RT-qPCR analysis demonstrated that intracellular miR-145 levels in hASCs transfected with miR-145 mimics was $~ 9,000$-fold higher when compared with cells transfected with the negative control $(\mathrm{P}=0.0001)$ (Fig. 2A). In addition, hASCs transfected with anti-miR-145 exhibited a $\sim 10$-fold lower level of miR-145 expression when compared with cells transfected with the control $(\mathrm{P}=0.029)$ (Fig. 2A). Following transfection, hASCs were cultured in medium supplemented with TGF- $\beta 1$ and BMP4 for seven days following starvation. The markers of SMCs were analyzed by RT-qPCR analysis. The mRNA levels of these markers were significantly increased following transfection with miR-145 mimic ( $\alpha$-SMA, $\mathrm{P}=0.0072$; SM22 $\alpha, \mathrm{P}=0.031$; calponin, $\mathrm{P}=0.038$; SM-MHC, $\mathrm{P}=0.0007$; Fig. 2B), whereas they were significantly decreased by anti-miR-145 treatment ( $\alpha$-SMA, $\mathrm{P}=0.0081 ; \mathrm{SM} 22 \alpha, \mathrm{P}=0.036$; calponin, $\mathrm{P}=0.015$; SM-MHC, $\mathrm{P}=0.035$; Fig. 2C), when compared with negative controls. In addition, the results demonstrated that smooth muscle-specific protein expression levels were significantly increased following transfection of miR-145 mimics ( $\alpha$-SMA,
$\mathrm{P}=0.024$; $\mathrm{SM} 22 \alpha, \mathrm{P}=0.345$; calponin, $\mathrm{P}=0.029$; $\mathrm{SM}-\mathrm{MHC}$, $\mathrm{P}=0.022)$, but were significantly decreased by anti-miR-145 $(\alpha-S M A, P=0.039$; SM22 $\alpha, \mathrm{P}=0.040$; calponin, $\mathrm{P}=0.026$; SM-MHC, $\mathrm{P}=0.011$; Fig. 2D), when compared with the negative controls. The results suggest that miR-145 may function as a positive regulator of smooth muscle differentiation in hASCs.

miR-145 targets KLF4. In order to understand the molecular mechanisms underlying miR-145-mediated regulation of SMC differentiation, potential targets of miR-145 that are implicated in SMC differentiation were identified, using miRNA target prediction algorithms, TargetScan and PicTar (30). The results demonstrated that MYOCD KlF-5, fascin, calcium/calmodulin kinase II- $\delta$ and adducin-3 are the predicted targets of miR-145. Among the predicted targets, KLF4 was identified as serving an established role in smooth muscle cell differentiation (20). Therefore, the expression level of KLF4 in SMC differentiation was examined in the present study. The results demonstrated that the mRNA level of KLF4 significantly decreased during treatment with TGF- $\beta 1$ and BMP4 at $5(\mathrm{P}=0.028)$ and $7(\mathrm{P}=0.016)$ days (Fig. 3A), which contrasted that of miR-145 expression (Fig. 1C). This suggests that miR-145 may suppress KLF4 expression during SMC differentiation. To verify that KLF4 is a functional target of miR-145, the wild-type 3'-UTR of KLF-4 (KLF4-3'-UTR-Wt) was cloned into a reporter plasmid. Co-transfection of the miR-145 mimic and KLF4-3'-UTR-Wt strongly decreased luciferase activity $(\mathrm{P}=0.033)$, whereas co-transfection of miR-control with KLF4-3'-UTR-Wt did not alter the luciferase activity (Fig. 3B). These results suggest that miR-145 inhibited the 3'-UTR of KLF4. A KLF4 

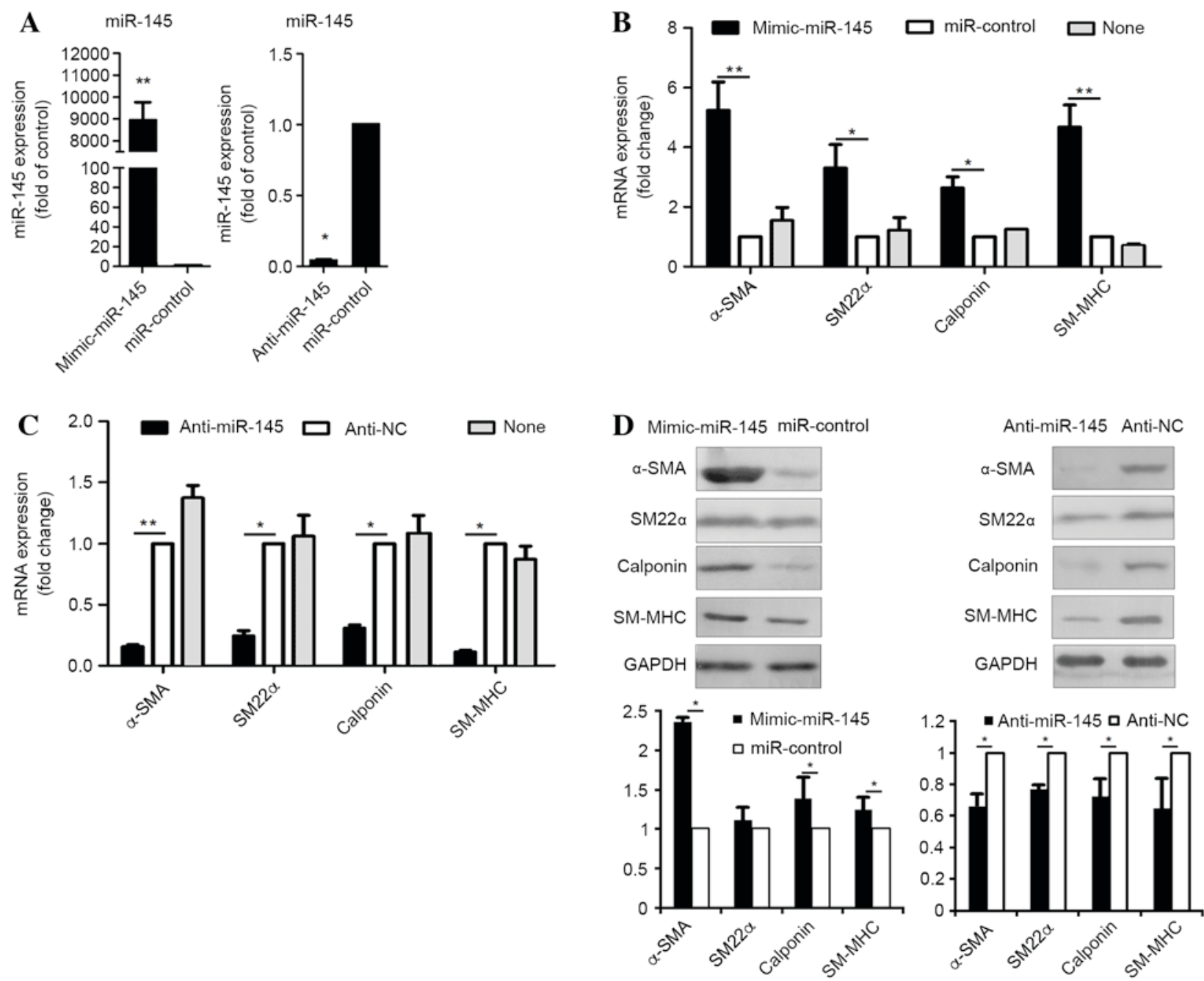

Figure 2. miR-145 promoted smooth muscle differentiation of hASCs. (A) miR-145 expression following transfection of miR-145 mimics or anti-miR-145 and treatment with TGF- $\beta$ and BMP4. The mRNA expression levels of smooth muscle markers following transfection with (B) mimic-miR-145 or miR-control and (C) anti-miR-145 or anti-NC. (D) Protein expression levels of smooth muscle markers in hASCs following transfection with miR-145 mimics or anti-miR-145. Data are presented as the mean \pm standard deviation $(n=3) .{ }^{*} \mathrm{P}<0.05$ and ${ }^{* *} \mathrm{P}<0.01$ vs. control or as indicated. miR-145, microRNA-145; hASCs, human adipose-derived stem cells; anti-NC, negative control of anti-miR-145; TGF- $\beta 1$, transforming growth factor- $\beta 1$; BMP4, bone morphogenetic protein $4 ; \alpha$-SMA, $\alpha$-smooth muscle actin; SM22 $\alpha$, smooth muscle protein-22 $\alpha$; SM-MHC, smooth muscle-myosin heavy chain.

luciferase reporter construct containing a predicted miR-145 binding site was mutated (KLF4-3'-UTR-Mut) to determine whether miR-145 targets KLF4 directly through the predicted binding site. This binding site is located at position 278-284 of the KLF4 3' UTR (14) Co-transfection of the miR-145 mimic and KLF4-3'-UTR-Mut did not alter the luciferase activity (Fig. 3B).

To investigate whether miR-145 may be able to downregulate KLF4 expression, the mRNA and protein levels were detected after hASCs were transfected with miR-145 mimics and inhibitors using RT-qPCR and western blotting analyses, respectively. The results indicated that the mRNA level of KLF4 was significantly decreased by miR-145 overexpression $(\mathrm{P}=0.029)$, whereas it was significantly increased by anti-miR-145 transfection $(\mathrm{P}=0.044)$, when compared with cells transfected with negative controls (Fig. 3C). In addition, the protein expression level of KLF4 was significantly higher in cells transfected with anti-miR-145 $(\mathrm{P}=0.017)$ and significantly lower in cells overexpressing miR-145 compared with the controls $(\mathrm{P}=0.031)$ (Fig. 3D). These results demonstrate that
miR-145 may downregulate KLF4 in hASCs treated with TGF- $\beta 1$ and BMP4.

KLF4 demonstrates negative effect on the smooth muscle differentiation of hASCs. To examine the role of KLF4 in the control of contractile gene expression, siRNA was used to knockdown KLF4 expression, thus mimicking the effect of miR-145 expression during induction by TGF- $\beta 1$ and BMP4. KLF4 protein expression was downregulated by $90 \%$ and mRNA expression was downregulated by $40 \%$, following transfection with KLF4 siRNA (si-KLF4) in hASCs (Fig. 4A and $\mathrm{B})$. Under these conditions, the levels of contractile proteins, $\alpha$-SMA, SM22 $\alpha$, calponin and SM-MHC were elevated, as determined by western blot analysis ( $\alpha$-SMA, $\mathrm{P}=0.039$; SM22 $\alpha, \mathrm{P}=0.152$; calponin, $\mathrm{P}=0.018$; SM-MHC, $\mathrm{P}=0.035$; Fig. 4C) and immunofluorescence staining (Fig. 4D), indicating that endogenous KLF4 constitutively represses the expression of contractile genes. These results provide further evidence of the inhibitory role of KLF4 in the induction of contractile genes by TGF- $\beta 1$ and BMP4. 
A

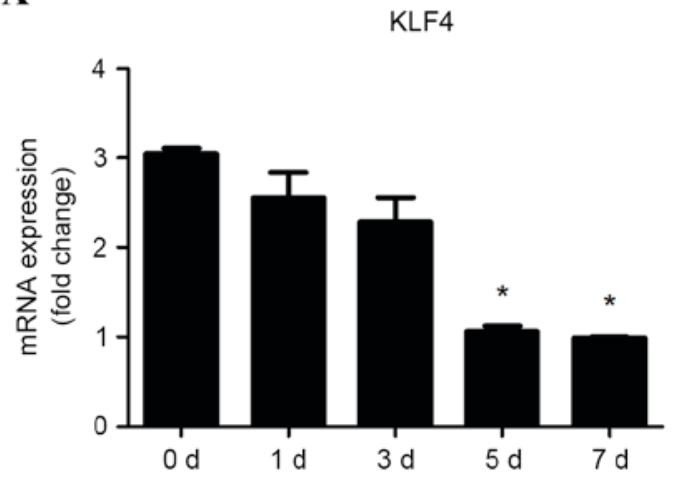

C

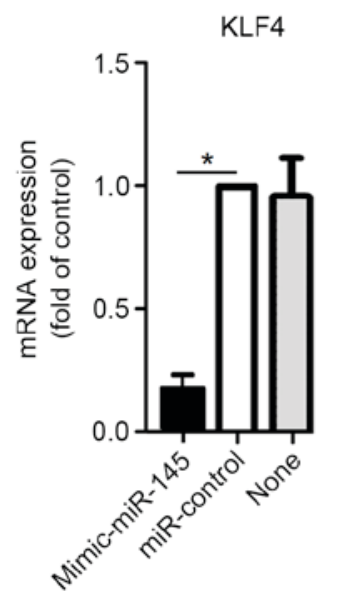

B

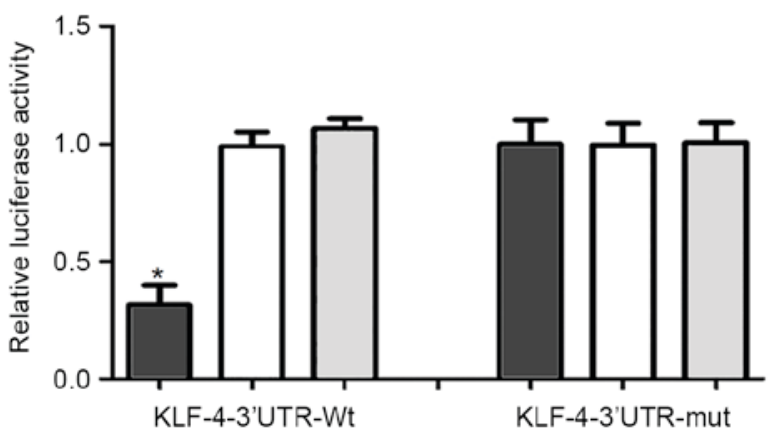

D

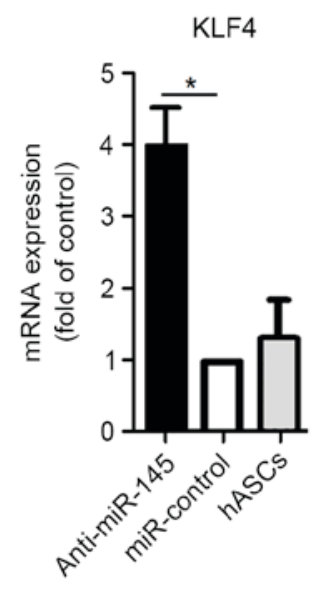

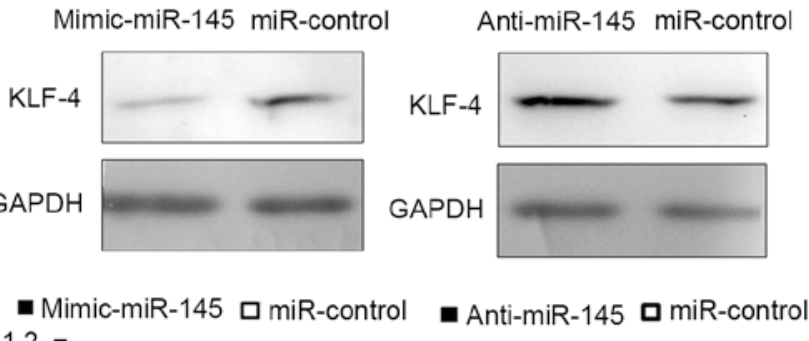
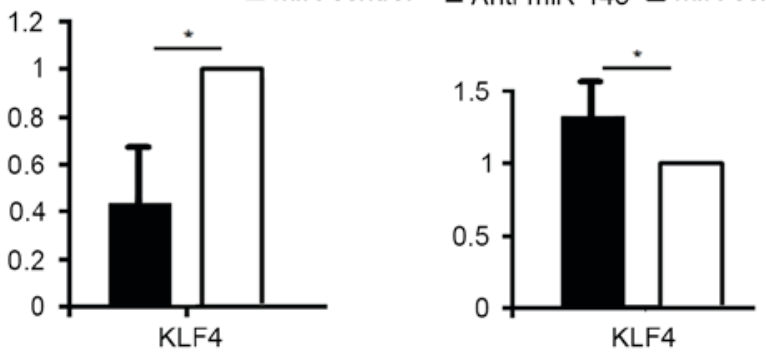

Figure 3. miR-145 targets KLF4. (A) KLF4 levels decreased gradually during differentiation of hASCs to smooth muscle cells (*P<0.05 vs. day 0). (B) Luciferase reporter assays validated KLF4 as a direct target of miR-145. The KLF4-3'UTR-Wt or the KLF4-3'UT R-Mut was transfected with the miR-145 mimic or miR-control into $293 \mathrm{~T}$ cells, followed by a dual-luciferase assay. Renilla/firefly luciferase ratios were calculated and further normalized to the empty vector control ('P<0.05 vs. KLF4-3'UTR-Wt + miR-control). The (C) mRNA and (D) protein expression levels of KLF4 when hASCs were induced after transfection with miR-145 mimics or anti-miR-145 ( ${ }^{*} \mathrm{P}<0.05$ vs. miR-control). Data are presented as the mean \pm standard deviation ( $\mathrm{n}=3$ ). miR-145, microRNA-145; KLF4, Krüppel-like factor 4; hASCs, human adipose-derived stem cells; KLF4-3'UTR-Wt, KLF4 reporter construct containing wild-type 3'-untranslated region; KLF4-3'UTR-Mut, KLF4 reporter construct containing a mutated predicted miR-145 binding site.

\section{Discussion}

SMCs exhibit a spectrum of phenotypes, ranging from the more differentiated 'contractile' state, in which high levels of SMC differentiation markers are expressed, to the less differentiated 'synthetic' state, in which SMC markers are downregulated $(15,31,32)$. Alterations in SMC differentiation states serve a major role in a number of cardiovascular diseases such as atherosclerosis, restenosis, hypertension and aneurysm (33). Therefore, an improved understanding of the molecular mechanisms that control SMC differentiation of ASCs is critical for enabling the development of novel strategies to prevent and treat these diseases.

miRNAs are small noncoding RNAs that regulate gene and protein expression by interacting with the 3'-UTR of the target mRNA sequences. This interaction results in mRNA degradation and/or inhibition or activation of protein translation (34). A number of miRNAs serve integral roles in smooth muscle development and contractile differentiation. The aim of the present study was to clarify the role of miRNAs in the SMC differentiation of hASCs. The results demonstrated that miR-145 promoted smooth muscle differentiation of hASCs. Firstly, ASCs were induced to differentiate to SMCs using TGF-b1 and BMP4, according to a previous study (13). The expression of smooth muscle contractile proteins and genes were increased following this induction. These results confirmed that ASCs successfully differentiated into SMCs. In addition to smooth muscle contractile proteins and genes, miR-145 expression was similarly upregulated during this process. ASCs were transfected with miR-145 mimics, antisense oligonucleotides and negative controls to investigate the effect of miR-145 on SMC differentiation of ASCs. The results indicated that miR-145 promotes smooth muscle contractile protein expression, while ASCs transfected with anti-miR-145 demonstrated the opposite effect. These findings are in accordance with earlier reports $(20,24,35)$.

To investigate the molecular mechanisms by which miR-145 regulates the smooth muscle differentiation of ASCs, potential target genes of miR-145 were investigated. One gene, KLF4, was hypothesized to be a target of miR-145 
A

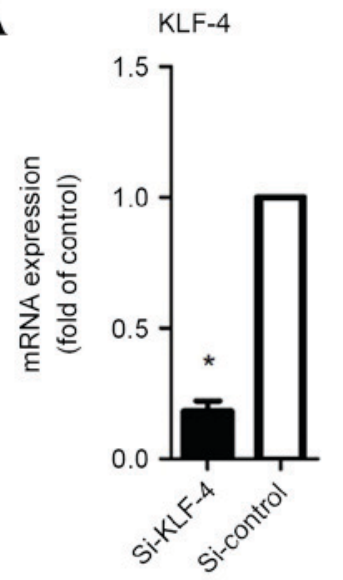

C

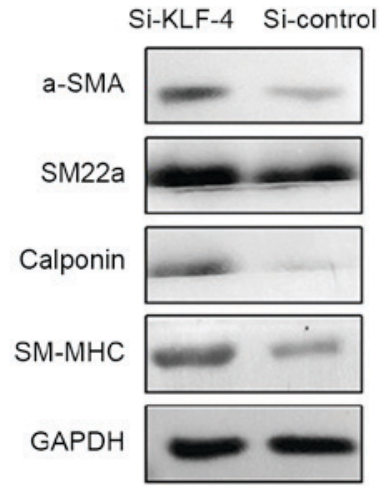

B

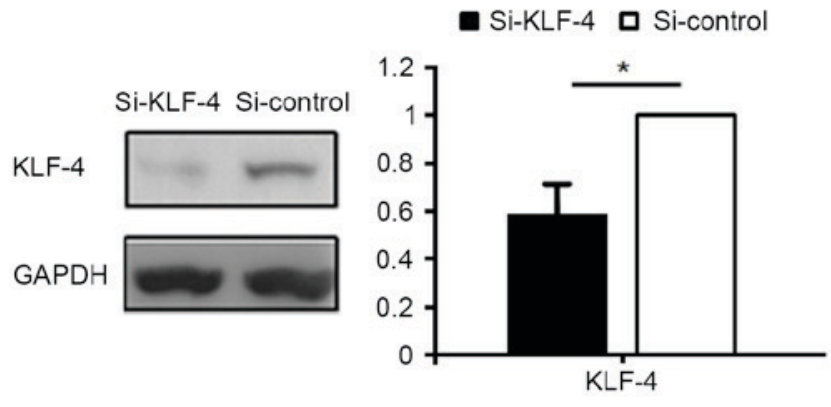

D

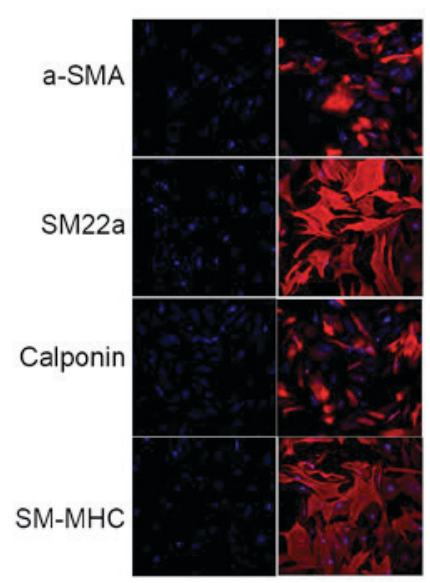

Figure 4. KLF4 represses the expression of smooth muscle contractile proteins and associated genes. The (A) mRNA and (B) protein expression levels of KLF4 following transfection with non-targeting siRNA (si-control) and siRNA targeting KLF4 (si-KLF-4). (C) Smooth muscle contractile protein expression following transfection with si-control and si-KLF4. (D) Following transfection with si-control and si-KLF4, cells were subject to immunofluorescence staining with antibodies against $\alpha$-SMA, SM22 $\alpha$, calponin and SM-MHC (red) and nuclear staining with DAPI (blue), respectively (magnification, x200). Data are presented as the mean \pm standard deviation $(\mathrm{n}=3)$. ${ }^{*} \mathrm{P}<0.05$ vs. si-control. KLF4, Krüppel-like factor 4 ; $\alpha$-SMD, $\alpha$-smooh muscle actin; siRNA, small interfering RNA; SM22 $\alpha$, smooth muscle protein-22 $\alpha$; SM-MHC, smooth muscle-myosin heavy chain.

using miRNA target prediction software. miR-145 has a target site in the 3'-UTR of KLF4 (20). Previous studies have demonstrated that KLF4 is involved in smooth muscle differentiation $(36,37)$.

In addition, miR-145 overexpression downregulated KLF4 at the protein and mRNA level, whereas functional inhibition of miR-145 by anti-miR-145 led to increased expression of KLF4. This suggested that miR-145 regulates KLF4 during smooth muscle differentiation. To examine the role of KLF4 in the control of contractile gene expression, siRNA was used to knockdown KLF4 expression, thus mimicking the effect of miR-145. Under these conditions, the expression levels of contractile proteins were dramatically elevated. This result was in accordance with previous reports (38-40) and indicated a negative effect of KLF4 on smooth muscle differentiation. KLF4 is a potent negative regulator of contractile genes, which functions through different mechanisms, including suppressing the transcriptional activation of the CArG box by SRF, MYOCD or MRTFs (19). In the current study, the functional significance of KLF4 downregulation by miR-145 during TGF- $\beta 1$ and BMP4-mediated induction of smooth muscle differentiation was investigated. The results established that basal expression of contractile genes increased following the siRNA-mediated knockdown of KLF4. By contrast, it was previously reported that, in rat aortic SMCs (rAoSMCs) (41) and mouse mesenchymal C3H10T1/2 cells (8), TGF- $\beta 1$ and BMP4 induced KLF4 expression at the same time points. Primary rAoSMCs treated with TGF- $\beta 1$ or BMP4 did not upregulate miR-145, and no repression of KLF4 was observed. Thus, the induction of miR-145 by TGF- $\beta 1$ and BMP4, and the subsequent suppression of KLF4, may be specific to human SMCs (20).

The present study aimed to predict additional targets of miR-145 alongside KLF4. The analysis demonstrated that miR-145 targets a wide range of factors, including MYOCD $\mathrm{KlF}-5$, fascin, calcium/calmodulin kinase II- $\delta$ and adducin-3. It could therefore be suggested that attenuation of the expression of these proteins may promote differentiation and repress the proliferation of SMCs. These targets are involved in a number of smooth muscle differentiation processes (24), and provide an example of how one miRNA may regulate several related or unrelated cellular processes. In addition to miR-145, miR-21, miR-221, miR-222 and miR-143 have established roles in VSMC differentiation. miR-21 promotes VSMC differentiation by negatively regulating the expression of programmed cell death protein 4 (42), whereas miR-221 and miR-222 promote VSMC proliferation by targeting the negative regulators of the cell cycle, p27 and p57 $(43,44)$. miR-143 
and miR-145-encoding genes are highly conserved, and are positioned in close proximity on mouse chromosome 18 and human chromosome 5 (24). Recently, miR-143 and miR-145, which are encoded as a gene cluster, were observed to target KLF4 and serve a critical role in regulation of the VSMC phenotype $(24,25,27)$. miR-143 or miR-145 VSMC gene knockout mice exhibited abnormal vascular tone and reduced contractile gene expression (24).

In conclusion, the current study demonstrated that miR-145 is a promoter of smooth muscle differentiation of hASCs induced by TGF- $\beta 1$ and BMP4. miR-145 targets the KLF4 gene, whose protein may function as a potent negative regulator of contractile gene expression. Overexpression of miR-145 suppressed KLF4 and promoted smooth muscle contractility induced by stem cells. There are additional mechanisms of smooth muscle differentiation, which will require further investigation. Further understanding of the molecular mechanisms would facilitate the treatment or prevention of smooth muscle-associated diseases.

\section{Acknowledgements}

The present study was financially supported by the National Natural Science Foundation of China (grant no. 81201204).

\section{References}

1. Rodríguez LV, Alfonso Z, Zhang R, Leung J, Wu B and Ignarro LJ Clonogenic multipotent stem cells in human adipose tissue differentiate into functional smooth muscle cells. Proc Natl Acad Sci USA 103: 12167-12172, 2006.

2. Lai JY, Yoon CY, Yoo JJ, Wulf T and Atala A: Phenotypic and functional characterization of in vivo tissue engineered smooth muscle from normal and pathological bladders. J Urol 168: 1853-1857, 2002

3. Silva GV, Litovsky S, Assad JA, Sousa AL, Martin BJ, Vela D, Coulter SC, Lin J, Ober J, Vaughn WK, et al: Mesenchymal stem cells differentiate into an endothelial phenotype, enhance vascular density, and improve heart function in a canine chronic ischemia model. Circulation 111: 150-156, 2005.

4. Bacou F, el Andalousi RB, Daussin PA, Micallef JP, Levin JM, Chammas M, Casteilla L, Reyne Y and Nougues J: Transplantation of adipose tissue-derived stromal cells increases mass and functional capacity of damaged skeletal muscle. Cell Transplant 13: 103-111, 2004.

5. Xu ZC, Zhang WJ, Li H, Cui L, Cen L, Zhou GD, Liu W and Cao Y: Engineering of an elastic large muscular vessel wall with pulsatile stimulation in bioreactor. Biomaterials 29 : 1464-1472, 2008.

6. Wang C, Cen L, Yin S, Liu Q, Liu W, Cao Y and Cui L: A small diameter elastic blood vessel wall prepared under pulsatile conditions from polyglycolic acid mesh and smooth muscle cells differentiated from adipose-derived stem cells. Biomaterials 31 : 621-630, 2010

7. Kern S, Eichler H, Stoeve J, Klüter H and Bieback K: Comparative analysis of mesenchymal stem cells from bone marrow, umbilical cord blood, or adipose tissue. Stem Cells 24: 1294-1301, 2006.

8. Cartwright MJ, Tchkonia T and Kirkland JL: Aging in adipocytes: Potential impact of inherent, depot-specific mechanisms. Exp Gerontol 42: 463-471, 2007.

9. Weinzierl K, Hemprich A and Frerich B: Bone engineering with adipose tissue derived stromal cells. J Craniomaxillofac Surg 34: 466-471, 2006.

10. Zuk PA, Zhu M, Ashjian P, De Ugarte DA, Huang JI, Mizuno H, Alfonso ZC, Fraser JK, Benhaim P and Hedrick MH: Human adipose tissue is a source of multipotent stem cells. Mol Biol Cell 13: 4279-4295, 2002.

11. Ashjian PA, Elbarbary AS, Edmonds B, DeUgarte D, Zhu M, Zuk PA, Lorenz HP, Benhaim P and Hedrick HK: In vitro differentiation of human processed lipoaspirate cells into early neural progenitors. Plast Reconstr Surg 111: 1922-1931, 2003.
12. Deslex S, Negrel R, Vannier C, Etienne J and Ailhaud G: Differentiation of human adipocyte precursors in a chemically defined serum-free medium. Int J Obes 11: 19-27, 1987.

13. Wang C, Yin S, Cen L, Liu Q, Liu W, Cao Y and Cui L: Differentiation of adipose-derived stem cells into contractile smooth muscle cells induced by transforming growth factor-beta1 and bone morphogenetic protein-4. Tissue Eng Part A 16: 1201-1213, 2010.

14. Lagna G, Ku MM, Nguyen PH, Neuman NA, Davis BN and Hata A: Control of phenotypic plasticity of smooth muscle cells by bone morphogenetic protein signaling through the myocardin-related transcription factors. J Biol Chem 282: 37244-37255, 2007.

15. Owens GK, Kumar MS and Wamhoff BR: Molecular regulation of vascular smooth muscle cell differentiation in development and disease. Physiol Rev 84: 767-801, 2004.

16. Yoshida T, Sinha S, Dandré F, Wamhoff BR, Hoofnagle MH, Kremer BE, Wang DZ, Olson EN and Owens GK: Myocardin is a key regulator of CArG-dependent transcription of multiple smooth muscle marker genes. Circ Res 92: 856-864, 2003.

17. Wang Z, Wang DZ, Hockemeyer D, McAnally J, Nordheim A and Olson EN: Myocardin and ternary complex factors compete for SRF to control smooth muscle gene expression. Nature 428: 185-189, 2004

18. Miano JM: Channeling to myocardin. Circ Res 95: 340-342, 2004.

19. Liu Y, Sinha S, McDonald OG, Shang Y, Hoofnagle MH and Owens GK: Kruppel-like factor 4 abrogates myocardin-induced activation of smooth muscle gene expression. J Biol Chem 280: 9719-9727, 2005

20. Davis-Dusenbery BN, Chan MC, Reno KE, Weisman AS, Layne MD, Lagna G and Hata A: Down-regulation of Kruppel-like factor-4 (KLF4) by microRNA-143/145 is critical for modulation of vascular smooth muscle cell phenotype by transforming growth factor-beta and bone morphogenetic protein 4. J Biol Chem 286: 28097-29110, 2011.

21. Alvarez-Garcia I and Miska EA: MicroRNA functions in animal development and human disease. Development 132: 4653-4662, 2005.

22. Hatfield SD, Shcherbata HR, Fischer KA, Nakahara K, Carthew RW and Ruohola-Baker H: Stem cell division is regulated by the microRNA pathway. Nature 435: 974-978, 2005.

23. Kanellopoulou C, Muljo SA, Kung AL, Ganesan S, Drapkin R, Jenuwein T, Livingston DM and Rajewsky K: Dicer deficient mouse embryonic stem cells are defective in differentiation and centromeric silencing. Genes Dev 19: 489-501, 2005.

24. Cordes KR, Sheehy NT, White MP, Berry EC, Morton SU, Muth AN, Lee TH, Miano JM, Ivey KN and Srivastava D: miR-145 and miR-143 regulate smooth muscle cell fate and plasticity. Nature 460: 705-710, 2009.

25. Cheng Y, Liu X, Yang J, Lin Y, Xu DZ, Lu Q, Deitch EA, Huo Y, Delphin ES and Zhang C: MicroRNA-145, a novel smooth muscle cell phenotypic marker and modulator, controls vascular neointimal lesion formation. Circ Res 105: 158-166, 2009.

26. Boettger T, Beetz N, Kostin S, Schneider J, Krüger M, Hein L and Braun T: Acquisition of the contractile phenotype by murine arterial smooth muscle cells depends on the Mir143/145 gene cluster. J Clin Invest 119: 2634-2647, 2009.

27. Xin M, Small EM, Sutherland LB, Qi X, McAnally J, Plato CF, Richardson JA, Bassel-Duby R and Olson EN: MicroRNAs miR-143 and miR-145 modulate cytoskeletal dynamics and responsiveness of smooth muscle cells to injury. Genes Dev 23: 2166-2178, 2009.

28. Kozomara A and Griffiths-Jones S: miRBase: Annotating high confidence microRNAs using deep sequencing data. Nucleic Acids Res 42 (Database Issue): D68-D73, 2014.

29. Livak KJ and Schmittgen TD: Analysis of relative gene expression data using real-time quantitative PCR and the 2(-Delta Delta C(T)) Method. Methods 25: 402-408, 2001.

30. Reczko M, Maragkakis M, Alexiou P, Papadopoulos GL and Hatzigeorgiou AG: Accurate microRNA target prediction using detailed binding site accessibility and machine learning on proteomics data. Front Genet 2: 103, 2012.

31. Sartore S, Chiavegato A, Faggin E, Franch R, Puato M, Ausoni S and Pauletto P: Contribution of adventitial fibroblasts to neointima formation and vascular remodeling: From innocent bystander to active participant. Circ Res 89: 1111-1121, 2001.

32. Walsh $\mathrm{K}$ and Takahashi A: Transcriptional regulation of vascular smooth muscle cell phenotype. Z Kardiol 90 (Suppl 3): S12-S16, 2001. 
33. Hirschi KK, Lai L, Belaguli NS, Dean DA, Schwartz RJ and Zimmer WE: Transforming growth factor-beta induction of smooth muscle cell phenotype requires transcriptional and post-transcriptional control of serum response factor. J Biol Chem 277: 6287-6295, 2002.

34. Bartel DP: MicroRNAs: Genomics, biogenesis, mechanism, and function. Cell 116: 281-297, 2004.

35. Ohnaka M, Marui A, Yamahara K, Minakata K, Yamazaki K, Kumagai M, Masumoto H, Tanaka S, Ikeda T and Sakata R: Effect of microRNA-145 to prevent vein graft disease in rabbits by regulation of smooth muscle cell phenotype. J Thorac Cardiovasc Surg 148: 676-682, 2014.

36. Salmon M, Gomez D, Greene E, Shankman L and Owens GK: Cooperative binding of KLF4, pELK-1 and HDAC2 to a G/C repressor element in the SM22 $\alpha$ promoter mediates transcriptional silencing during SMC phenotypic switching in vivo. Circ Res 111: 685-696, 2012.

37. Yoshida T, Kaestner KH and Owens GK: Conditional deletion of Krüppel-like factor 4 delays downregulation of smooth muscle cell differentiation markers but accelerates neointimal formation following vascular injury. Circ Res 102: 1548-1557, 2008.

38. Cherepanova OA, Pidkovka NA, Sarmento OF, Yoshida T, Gan Q, Adiguzel E, Bendeck MP, Berliner J, Leitinger N and Owens GK: Oxidized phospholipids induce type VIII collagen expression and vascular smooth muscle cell migration. Circ Res 104: 609-618, 2009.
39. Pidkovka NA, Cherepanova OA, Yoshida T, Alexander MR, Deaton RA, Thomas JA, Leitinger N and Owens GK: Oxidized phospholipids induce phenotypic switching of vascular smooth muscle cells in vitro and in vivo. Circ Res 101: 792-801, 2007.

40. Liu Y, Sinha S and Owens G: A transforming growth factor-beta control element required for SM alpha-actin expression in vivo also partially mediates GKLF-dependent transcriptional repression. J Biol Chem 278: 48004-48011, 2003.

41. Li HX, Han M, Bernier M, Zheng B, Sun SG, Su M, Zhang R, Fu JR and Wen JK: Krüppel-like factor 4 promotes differentiation by transforming growth factor-beta receptor-mediated Smad and p38 MAPK signaling in vascular smooth muscle cells. J Biol Chem 285: 17846-17856, 2010.

42. Davis BN, Hilyard AC, Lagna G and Hata A: SMAD proteins control DROSHA-mediated microRNA maturation. Nature 454: 56-61, 2008.

43. Davis BN, Hilyard AC, Nguyen PH, Lagna G and Hata A: Induction of microRNA-221 by platelet-derived growth factor signaling is critical for modulation of vascular smooth muscle phenotype. J Biol Chem 284: 3728-3738, 2009.

44. Liu X, Cheng Y, Zhang S, Lin Y, Yang J and Zhang C: A necessary role of miR-221 and miR-222 in vascular smooth muscle cell proliferation and neointimal hyperplasia. Circ Res 104: 476-487, 2009. 